COMMENT. Patients with epilepsy of driving age may be counseled that factors known to reduce the risk of seizure-related automobile accidents include optimal AED therapy with long seizure-free intervals, reliable auras, and few prior nonseizure-related accidents. The authors comment that if the 50 patients who had crashed had honored a 12-month legally defined seizure-free restriction, $74 \%$ of crashes might have been averted. A longer seizure-free restriction on driving could lead to poor compliance and a higher risk of crashes. Patients whose medication was closely monitored and optimized showed an $89 \%$ reduction in risk of crashes.

Berg AT and Engel J Jr, in an editorial, discuss "Restricted driving for people with epilepsy." (Neurology April 1999;52:1306-1307). The minimum seizurefree interval of 12 months is the strongest predictor of avoidance of motor vehicle accidents for patients with epilepsy. This 1-year restriction on driving is generally appropriate and is mandated by the European Union Association of International Bureau for Epilepsy. A US consensus development workshop opinion, approved by the AAN, AES, and EFA, recommends a minimum 3-month seizure-free interval, recognizing the importance of the neurologist's input and need to consider mitigating factors. The study by Krauss et al provides evidence for some of the factors that may permit reduction of the 1-year restriction in certain individuals: eg reliable aura, accident-free prior driving record, and close AED monitoring and compliance.

\title{
HEREDO-DEGENERATIVE DISEASE
}

\section{INFANTILE NEUROAXONAL DYSTROPHY: DIAGNOSTIC CRITERIA}

The diagnostic significance of clinical, neurophysiologic, and neuroradiologic findings in 13 patients with infantile neuroaxonal dystrophy (INAD) was evaluated at the National Neurological Institute, Milan, Italy. Symptoms and signs of psychomotor regression presented between 6 months and 2 years of age. The clinical course was typical in 9 patients, with rapid motor and mental deterioration: it was atypical in 4 showing a slower rate of progression. Late neurologic features included optic atrophy and vision failure in 8 , nystagmus in 3 , and spastic or areflexic tetraparesis with axial hypotonia and distal contractures in all patients. EMG signs of chronic denervation, EEG fast activity, and abnormal visual evoked potentials occurred in all patients during the course of the disease. NCS became abnormal and consistent with axonal sensorimotor peripheral neuropathy in 9. All patients had histologic evidence of spheroids on skin biopsy. Characteristic MRI abnormalities were cerebellar atrophy, first involving the inferior vermis and later the cortex, with hyperintense signal on T2-weighted images, and hypointensity in the pallida and substantia nigra. a-N-acetyl-galactosaminidase leukocyte activity was normal in 10 patients tested. (Nardocci N, Zorzi G, Farina L et al. Infantile neuroaxonal dystrophy. Clinical spectrum and diagnostic criteria. Neurology April 1999;52:1472-1478). (Reprints: Dr Nardo Nardocci, Department of Child Neurology, National Neurological Institute "Carlo Besta," Via Celoria 11, 20133 Milan, Italy).

COMMENT. The diagnostic criteria for INAD are as follows: 1) spheroids on skin biopsy; 2) onset before 3 years; 3) psychomotor deterioration, with symmetric pyramidal tract signs and truncal hypotonia; and 4) progression to spastic tetraparesis, blindness, and dementia by the age of 4 years. Atypical cases may occur, with slower progression beginning between 7 and 12 years after a stable course resembling static encephalopathy, and hypotonic-areflexic 
tetraparesis with cerebellar signs, but without spasticity and signs of pyramidal dysfunction. The disease has greater clinical vartiability than previously recognized. MRI findings can be helpful in diagnosis, with early involvement and atrophy of the cerebellar vermis. The pallidal involvement in 2 of the above series suggests an overlap with the radiologic findings in Hallervorden-Spatz disease. A biochemical or molecular marker has not been uncovered.

\section{NEUROMUSCULAR DISEASES}

\section{OUTCOME IN SEVERE GUILLAIN-BARRE SYNDROME}

The effect of various therapies on duration of illness in children with severe Guillain-Barre syndrome (GBS) was evaluated at CHMC, Seattle, WA. Of 26 children treated in two contiguous 8-year periods, 12 received supportive care alone (SC), and 14 were treated with SC plus either plasma exchange (PE), 6 cases, or intravenous immunoglobulin (IVIg), 8 cases. Recovery to score 2 (able to walk $5 \mathrm{~m}$ without support) was similar in the SC and IVIg groups, with a nonsignificant trend toward longer recovery times in the PE group. The addition of PE or IVIg did not improve outcome or shorten duration of illness compared with SC alone. Full recovery occurred in almost all patients within 6 months of disease onset. (Graf WD, Katz JS, Eder DN, Smith AJ, Chun MR. Outcome in severe Guillain-Barre syndrome after immunotherapy or supportive care. Neurology April 1999;52:14941497). (Respond: WD Graf MD, Division of Child Neurology, CHMC, 4800 Sandpoint Way, Seattle, WA 98105).

COMMENT. Immunotherapy in severe pediatric GBS does not improve outcome or shorten duration of illness compared with supportive care alone and may be less effective than in adult cases of GBS.

\section{BETHLEM MYOPATHY}

The natural course of Bethlem myopathy in five previously published kindreds and two novel pedigrees was investigated, with attention to the mode of onset in 23 children and the progression of weakness in 36 adult patients followed at the Academic Medical Center, Amsterdam, The Netherlands. Onset was characterized by diminished fetal movements, neonatal hypotonia and congenital contractures including torticollis, nearly all children exhibiting weakness or contractures and slightly delayed milestones during the first 2 years of life. Symptoms became more evident at 5 years of age, with worsening of contractures and weakness during childhood, followed by relative recovery during puberty. During early adult life, many patients were nearly asymptomatic except for contractures. From middle age onwards, the contractures remained constant but weakness and incapacity showed slow but ongoing progression into adulthood, more than two-thirds of patients over 50 years of age requiring a wheelchair. (Jobsis GJ, Boers JM, Barth PG, de Visser M. Bethlem myopathy: a slowly progressive congenital muscular dystrophy with contractures. Brain April 1999;122:649-655). (Respond: Dr GJ Jobsis, Department of Neurology, (H2-214), Academic Medical Center, PO Box 22700, 1100 DE Amsterdam, The Netherlands).

COMMENT. In 1976, Bethlem and van Wijngaarden described three families with an early-onset benign autosomal dominant myopathy with contractures. Contractures involved fingers, wrists, elbows, shoulders, knees and hips. Weakness was mild, affecting proximal and extensor muscles, with only limited functional impairment, even in old age. Cranial and cardiac muscles were not 